\title{
New Procedures of Estimating Proportion and Sensitivity Using Randomized Response in a Dichotomous Finite Population
}

Tanveer A. Tarray

School of Studies in Statistics, Vikram University Ujjain - M.P. - India., tanveerstat@gmail.com

Housila P. Singh

School of Studies in Statistics, Vikram University Ujjain, M.P., India, hpsujn@gmail.com

Follow this and additional works at: http://digitalcommons.wayne.edu/jmasm

Part of the Applied Statistics Commons, Social and Behavioral Sciences Commons, and the Statistical Theory Commons

\section{Recommended Citation}

Tarray, Tanveer A. and Singh, Housila P. (2016) "New Procedures of Estimating Proportion and Sensitivity Using Randomized Response in a Dichotomous Finite Population," Journal of Modern Applied Statistical Methods: Vol. 15 : Iss. 1 , Article 32. DOI: $10.22237 /$ jmasm/1462077060

Available at: http://digitalcommons.wayne.edu/jmasm/vol15/iss1/32 


\section{New Procedures of Estimating Proportion and Sensitivity Using Randomized Response in a Dichotomous Finite Population}

\section{Cover Page Footnote}

The authors are thankful to the Editor - in- Chief, and to the anonymous learned referee for his valuable suggestions regarding improvement of the paper. 


\section{New Procedures of Estimating Proportion and Sensitivity Using Randomized Response in a Dichotomous Finite Population}

\author{
Tanveer A. Tarray \\ Vikram University \\ Ujjain, M. P., India
}

\author{
Housila P. Singh \\ Vikram University \\ Ujjain, M. P., India
}

The problem of estimating the population proportion possessing a sensitive attribute using simple random sampling with replacement (SRSWR) is advocated. Two new procedures are proposed. The suggested models are more efficient than the Huang (2004) randomized response technique under some realistic conditions. Numerical and graphic illustrations are given.

Keywords: Randomized response technique, direct response, estimation of proportion, privacy of respondents, sensitive characteristics

\section{Introduction}

Socioeconomic investigations often relate to certain personal features that people desire to hide from others in comprehensive inquiries, detailed questionnaires include numerous items. Direct questioning of respondents about them is likely to result either in non-response or in a deliberately incorrect answer. Social stigma and fear of reprisals often lead respondents to give biased, misleading, or even erroneous responses when approached with a direct response (DR) survey method. Even for the reason of merely being unwilling to reveal secrets to strangers, many individuals attempt to avoid certain questions put to them by interviewers.

Consider a dichotomous population in which every person belongs to either a sensitive group A or to the non-sensitive complement $A^{c}$. The aim is to estimate $\pi$, the population proportion of individuals who are members of A. To do so, a simple random sample of size $n$ is drawn from the population with replacement. Let $T$ be the probability that the respondents belong to A report the truth. The respondents

Dr. Tarray is affiliated with the Department of Computer Science and Engineering. Email him at: tanveerstat@gmail.com.Dr. Singh is affiliated with the School of Studies in Statistics. 


\section{TARRAY \& SINGH}

belonging to the non-sensitive group $\mathrm{A}^{\mathrm{c}}$ have no reason to tell a lie. For a DR survey of size $n$, the interviewee is asked if they are a member of $\mathrm{A}$. Then we have a direct estimator

$$
\hat{\pi}_{D}=\frac{\sum_{i=1}^{n} X_{i}}{n}
$$

with mean square error (MSE) given by

$$
\operatorname{MSE}\left(\hat{\pi}_{D}\right)=\frac{\theta_{D}\left(1-\theta_{D}\right)}{n}+\pi^{2}(1-T)^{2}
$$

where $X_{i}=1(0)$ if the $i^{\text {th }}$ interviewee responds Yes (No) and $\theta_{D}=\pi T$.

To procure reliable sample data for the population proportion of the respondents belonging to the sensitive group A, Warner (1965) proposed an ingenious procedure called Warner's randomized response technique. This pioneering work led to modification and developments in several directions; for instance, see Fox and Tracy (1986), Mangat and Singh (1990), Mangat (1994), Mahmood, Singh, and Horn (1998), Chua and Tsui (2000), Sing, Singh, and Mangat (2000), Chang and Huang (2001), Huang (2004), Chang, Wang, and Huang (2004a, b) and Singh and Tarray (2012, 2013a, b, c, 2014a, b, c, d, e).

Huang (2004) pointed out there are many variants of the randomized response technique in the literature, but most do not dwell on the fundamental question: whether or not the issues considered in the survey should be regarded as sensitive, meaning that there is a need for a randomized response procedure rather than a direct response procedure. In general, the probability $T$ is a measure instrument of the sensitivity (see Huang, 2004). It has a primary use in appraising the efficiency of different survey plans. One may use a simple formula for ascertaining whether a randomized response technique is beneficial in efficiency relative to a DR scheme. However, the probability $T$ is unknown in actual practice. To overcome such a difficulty, Chang and Huang (2001), Huang (2004), and Chang et al. (2004a) have suggested alternative survey strategies which make it possible to estimate the unknown parameters $\pi$ and $T$ simultaneously. Two alternatives to Huang's (2004) randomized response model, based on Singh (1993) models, are proposed. 


\section{A Brief Review of Randomized Response Models}

\section{Warner's Models}

In order to improve respondent cooperation and to encourage honest response, Warner (1965) proposed the following procedure, known as a randomized response technique (RRT). Instead of a DR procedure, a randomization device is used to gather sample information consisting of one of two statements:

(i) "I am a member of group A"

(ii) "I am not a member of group A"

with probabilities $P$ and $(1-P)$ respectively. Following this device, the respondent selects a statement unobserved by the interviewer, and then simply gives a "Yes" or "No" answer in a random sample of $n$ respondents. By the method of moments, Warner obtained an unbiased estimator of the population proportion $\pi$ possessing the sensitive attribute A:

$$
\hat{\pi}_{W}=\frac{\bar{Y}-(1-P)}{2 P-1}, \quad P \neq \frac{1}{2},
$$

where $Y_{i}=1(0)$ if the $i^{\text {th }}$ respond answers Yes (No) and $\bar{Y}=\frac{1}{2} \sum_{i=1}^{n} y_{i}$. The variance of $\hat{\pi}_{W}$ is given by

$$
\begin{aligned}
\mathrm{V}\left(\hat{\pi}_{W}\right) & =\frac{\theta_{W}\left(1-\theta_{W}\right)}{n(2 P-1)^{2}}, \\
& =\frac{\pi(1-\pi)}{n}+\frac{P(1-P)}{n(2 P-1)^{2}},
\end{aligned}
$$

where $\theta_{W}=\pi P+(1-\pi)(1-P)$.

\section{Singh Models}

Singh (1993) developed two randomized response techniques named RRT1 and RRT2 which are given below. 


\section{TARRAY \& SINGH}

RRT1: In this procedure, each interviewee in A with replacement simple random sample of size $n$ is provided with one randomized response device. It consists of the statement "I belong to the sensitive group" with known probability $P$, exactly the same probability as used by Warner (1965) and the statement "Yes" with probability $(1-P)$. The interviewee is instructed to use the device and report "Yes" or "No" for the random outcome of the sensitive statement according to his/her actual status. Otherwise, he is simply to report the "Yes" statement observed on the randomized response device. The whole procedure is completed by the respondent, unobserved by the interviewer. Then $\theta_{1}$, the probability of a "Yes" answer in the population, is

$$
\theta_{S 1}=P \pi+(1-P)
$$

An unbiased estimator of $\pi$ due to Singh (1993) is given by

$$
\hat{\pi}_{S 1}=\frac{\hat{\theta}_{S 1}-(1-P)}{P},
$$

where $\hat{\theta}_{S 1}$ is the proportion of "Yes" answers in the sample of size $n$.

The variance of the estimator $\hat{\pi}_{S 1}$ is given by

$$
\mathrm{V}\left(\hat{\pi}_{S 1}\right)=\frac{\pi(1-\pi)}{n}+\frac{(1-P)(1-\pi)}{n P} .
$$

RRT2: $\quad$ This procedure is exactly like RRT1 except for a change in probabilities on the randomized response device, i.e., the probabilities for the "sensitive" statement and "Yes" statement have been interchanged. The probability of a "Yes" response is then

$$
\hat{\pi}_{S 2}=\frac{\hat{\theta}_{S 2}-P}{(1-P)},
$$

with variance is given by 


$$
\mathrm{V}\left(\hat{\pi}_{S 2}\right)=\frac{\pi(1-\pi)}{n}+\frac{P(1-\pi)}{n(1-P)}
$$

Huang (2004) showed that his procedure resulted in better performance as compared to the Warner (1965) and Chang and Huang (2001) procedures.

\section{Huang Model}

In this procedure, a simple random sample of size $n$ is drawn with replacement from a finite population. The sampled individuals are required to reply to a direct query as to whether or not they belong to A. When answering "No", the respondent is provided with a randomization device consisting of two statements:

(i) "I am a member of A"

(ii) "I am not a member of A"

with probabilities $P$ and $(1-P)$, respectively.

It is assumed that the respondents belonging to A give totally honest responses under the randomized response procedure, but with probability $T$ following the usual direct response procedure. The probability of a "Yes" response in the direct response procedure is given by

$$
\theta_{1}=\pi T
$$

and in the randomized response procedure by

$$
\theta_{2}=P \pi(1-T)+(1-P)(1-\pi)=(2 P-1) \pi-P \pi T+(1-P)
$$

Huang (2004) suggested the following estimators of $\pi$ and $T$ respectively as

$$
\hat{\pi}_{H}=\frac{\left[P \hat{\theta}_{1}+\hat{\theta}_{2}-(1-P)\right]}{(2 P-1)}
$$

and 


$$
\hat{T}_{H}=\frac{(2 P-1) \hat{\theta}_{1}}{\left[P \hat{\theta}_{1}+\hat{\theta}_{2}-(1-P)\right]},
$$

where $\hat{\theta}_{j}$, the observed proportion of "Yes" answers, is the binomial random variable with parameters $n$ and $\theta_{j}, j=1,2$. Huang (2004) obtained the variance of $\hat{\pi}_{H}$ as

$$
\mathrm{V}\left(\hat{\pi}_{H}\right)=\frac{\pi(1-\pi)}{n}+\frac{P(1-P)(1-\pi T)}{n(2 P-1)^{2}}
$$

and the MSE of the estimator $\hat{T}_{H}$, up to terms of order $\mathrm{O}\left(n^{-1}\right)$, as

$$
\operatorname{MSE}\left(\hat{T}_{H}\right)=\frac{T(1-T)}{n \pi}+\frac{P(1-P) T^{2}(1-\pi T)}{n(2 P-1)^{2} \pi^{2}}
$$

\section{Proposed Procedures}

\section{HRRT1}

In this procedure, a simple random sample of size $n$ is drawn with replacement from a finite population. The sampled individuals are instructed to answer a direct query as to whether or not he/she belongs A. When answering "No", the respondent is provided with a randomization device. It consists of the statement "I belong to the sensitive group" with known probability $P$, exactly the same probability as used by Warner (1965), and the statement "Yes" with probability $(1-P)$ (Singh 1993, p. 68). The interviewee is instructed to use the device and report "Yes" or "No" for the random outcome of the sensitive statement according to his/her actual status. Otherwise, they are simply to report the "Yes" statement observed on the randomized response device. The whole procedure is completed by the respondent, unobserved by the interviewer. Then $\theta_{t 1}$, the probability of a "Yes" answer in the population, is

$$
\theta_{t 1}=\pi T \Rightarrow T=\frac{\theta_{t 1}}{\pi}
$$




\section{NEW PROCEDURES OF ESTIMATING PROPORTION USING RRT}

And, adopting the randomized response procedure, the respondent gives totally honest responses under the randomized response procedure by

$$
\theta_{t 1}=P \pi(1-T)+(1-P) \Rightarrow \pi=\frac{\left[P \theta_{t 1}+\theta_{t 2}-(1-P)\right]}{P}
$$

Thus the proposed estimators of $\pi$ and $T$ are given by

$$
\hat{\pi}_{a 1}=\frac{\left[P \hat{\theta}_{t 1}+\hat{\theta}_{t 1}-(1-P)\right]}{P}
$$

and

$$
\hat{T}_{1}=\frac{P \hat{\theta}_{t 1}}{\left[P \hat{\theta}_{t 1}+\hat{\theta}_{t 2}-(1-P)\right]}
$$

respectively, where $\hat{\theta} t j$, the observed proportion of "Yes" answers, is the binomial random variable with parameters $n$ and $\theta_{t j}, j=1,2$. The principal properties of the estimator $\hat{\pi}_{a 1}$ are outlined in the following theorem:

Theorem 1. The estimator $\hat{\pi}_{a 1}$ is unbiased with the variance given by

$$
\mathrm{V}\left(\hat{\pi}_{a 1}\right)=\frac{\pi(1-\pi)}{n}+\frac{(1-P)(1-\pi-\pi T)}{n P}
$$

Proof. The unbiasedness follows from $\mathrm{E}\left(\hat{\theta}_{t j}\right)=\theta_{t j}, j=1,2$. The variance of the estimator $\hat{\pi}_{a 1}$ can be obtained as follows: 


\section{TARRAY \& SINGH}

$$
\begin{aligned}
\mathrm{V}\left(\hat{\pi}_{a 1}\right) & =\frac{1}{P^{2}}\left[P^{2} \mathrm{~V}\left(\hat{\theta}_{t 1}\right)+\mathrm{V}\left(\hat{\theta}_{t 2}\right)+2 P \operatorname{cov}\left(\hat{\theta}_{t 1}, \hat{\theta}_{t 2}\right)\right] \\
& =\frac{1}{P^{2}}\left[\frac{P^{2} \theta_{t 1}\left(1-\theta_{t 1}\right)}{n}+\frac{\theta_{t 2}\left(1-\theta_{t 2}\right)}{n}-\frac{2 P \theta_{t 1} \theta_{t 2}}{n}\right] \\
& =\frac{1}{n P^{2}}\left[P^{2} \theta_{t 1}+\theta_{t 2}-\left(P^{2} \theta_{t 1}^{2}+\theta_{t 2}^{2}+2 P \theta_{t 1} \theta_{t 2}\right)\right] \\
& =\frac{1}{n P^{2}}\left[P^{2} \theta_{t 1}+\theta_{t 2}-\left(P \theta_{t 1}+\theta_{t 2}\right)^{2}\right] \\
& =\frac{1}{n P^{2}}\left[P^{2} \pi(1-\pi)+P(1-P)(1-\pi-\pi T)\right] \\
& =\frac{1}{n}\left[\pi(1-\pi)+\frac{(1-P)(1-\pi-\pi T)}{P}\right]
\end{aligned}
$$

Hence the theorem.

An unbiased estimator of the variance $\mathrm{V}\left(\hat{\pi}_{a 1}\right)$ can easily be obtained, which is given as follows:

Theorem 2. The unbiased estimator of $\mathrm{V}\left(\hat{\pi}_{a 1}\right)$ is given by

$$
\mathrm{V}\left(\hat{\pi}_{a 1}\right)=\frac{\hat{\pi}_{a 1}\left(1-\hat{\pi}_{a 1}\right)}{n-1}+\frac{(1-P)\left(1-\hat{\pi}_{a 1}-\hat{\theta}_{a 1}\right)}{(n-1) P}
$$

To form an idea about the sampling fluctuation of the direct estimator $\hat{\pi}_{D}$ from the sample itself, one has to develop an estimator of $\operatorname{MSE}\left(\hat{\pi}_{D}\right)$. In fact, with the help of the proposed procedure, one can find an unbiased estimator of the MSE of $\hat{\pi}_{a 1}$, which is presented in the following theorem:

Theorem 3. The unbiased estimator of the MSE of $\hat{\pi}_{D}$ is given by 


$$
\begin{aligned}
\operatorname{MSE}\left(\hat{\pi}_{D}\right) & =\left(\hat{\theta}_{t 1}-\hat{\pi}_{a 1}\right)^{2}+\frac{2 \hat{\theta}_{t 1}}{(n-1)}\left[1-\hat{\pi}_{a 1}-\frac{(1-P)}{p}\right]-\hat{\mathrm{V}}\left(\hat{\pi}_{a 1}\right) \\
& =\left(\hat{\theta}_{t 1}-\hat{\pi}_{a 1}\right)^{2}+\frac{2 \hat{\theta}_{t 1}}{(n-1) P}\left[P-P \hat{\theta}_{t 1}-\hat{\theta}_{t 2}\right]-\hat{\mathrm{V}}\left(\hat{\pi}_{a 1}\right)
\end{aligned}
$$

Proof. The proof is straightforward and is therefore omitted.

To obtain the bias and MSE of the estimator $\hat{T}$, we write $d_{1}=P \hat{\theta}_{1}$ and $d_{2}=\left[P \hat{\theta}_{1}+\hat{\theta}_{2}-(1-P)\right]$, and it follows that $\mathrm{E}\left(d_{1}\right)=P \pi T$ and $\mathrm{E}\left(d_{2}\right)=\pi P$. The estimator $\hat{T}$ can then be represented as $\hat{T}=d_{1} / d_{2}$, and we have $T=\mathrm{E}\left(d_{1}\right) / \mathrm{E}\left(d_{2}\right)$. Furthermore, we define the following quantities:

$$
e_{1}=\frac{d_{1}-\mathrm{E}\left(d_{1}\right)}{\mathrm{E}\left(d_{1}\right)}
$$

and

$$
e_{2}=\frac{d_{2}-\mathrm{E}\left(d_{2}\right)}{\mathrm{E}\left(d_{2}\right)}
$$

assuming that $\left|e_{1}\right|<1$ so that the function $\left(1+\mathrm{e}_{2}\right)^{-1}$ can be validly expanded as a power series. It can be easily proved that

$$
\begin{aligned}
\mathrm{E}\left(e_{1}^{2}\right) & =\frac{\theta_{t 1}\left(1-\theta_{t 1}\right)}{n \theta_{t 1}^{2}}=\frac{\left(1-\theta_{t 1}\right)}{n \theta_{t 1}}=\frac{\theta_{t 1}\left(1-\theta_{t 1}\right)}{n \pi^{2} T^{2}}, \\
\mathrm{E}\left(e_{2}^{2}\right) & =\frac{P^{2} \theta_{t 1}\left(1-\theta_{t 1}\right)+\theta_{t 2}\left(1-\theta_{t 2}\right)-2 P \theta_{t 1} \theta_{t 2}}{n P^{2} \pi^{2}}, \\
\mathrm{E}\left(e_{1} e_{2}\right) & =\frac{P \theta_{t 1}\left(1-\theta_{t 1}\right)-\theta_{t 1} \theta_{t 2}}{n P \pi^{2} T}
\end{aligned}
$$

and the estimation error of the estimator $\hat{T}_{1}$ can be expressed as 


\section{TARRAY \& SINGH}

$$
\begin{aligned}
\hat{T}_{1}-T & =\frac{P \theta_{t 1}\left(1+e_{1}\right)-\theta_{t 1} \theta_{t 2}}{P \pi\left(1+e_{2}\right)}-T \\
& =\frac{P \pi T}{P \pi}\left(1+e_{1}\right)\left(1+e_{2}\right)^{-1}-T \\
& =T\left(1+e_{1}\right)\left(1+e_{2}\right)^{-1}-T \\
\hat{T}_{1}-T & =T\left(e_{1}-e_{2}\right)+\mathrm{o}_{P}\left(n^{-1 / 2}\right)
\end{aligned}
$$

We then state the following theorem:

Theorem 4. The MSE of the estimator $\hat{T}_{1}$, up to terms of order $\mathrm{o}\left(n^{-1}\right)$, is given by

$$
\operatorname{MSE}\left(\hat{T}_{1}\right)=\frac{T(1-T)}{n \pi}+\frac{(1-P) T^{2}(1+\pi-\pi T)}{n P \pi^{2}}
$$

Proof. Consider

$$
\begin{aligned}
\operatorname{MSE}\left(\hat{T}_{1}\right) & =\mathrm{E}\left(\hat{T}_{1}-T\right)^{2} \\
& \cong T^{2}\left[\mathrm{E}\left(e_{1}^{2}\right)-2 \mathrm{E}\left(e_{1} e_{2}\right)+\mathrm{E}\left(e_{2}^{2}\right)\right] \\
& =\frac{T^{2}}{n}\left[\begin{array}{l}
\frac{\theta_{t 1}\left(1-\theta_{t 1}\right)}{\pi^{2} T^{2}}-\frac{2\left[P \theta_{t 1}\left(1-\theta_{t 1}\right)-\theta_{t 1} \theta_{t 2}\right]}{P \pi^{2} T^{2}} \\
\left.\quad+\frac{P^{2} \theta_{t 1}\left(1-\theta_{t 1}\right)+\theta_{t 2}\left(1-\theta_{t 2}\right)-2 P \theta_{t 1} \theta_{t 2}}{P^{2} \pi^{2}}\right]
\end{array}\right] \\
& =\frac{T^{2}}{n \pi^{2}}\left[\frac{\theta_{t 1}\left(1-\theta_{t 1}\right)(1-T)^{2}}{T^{2}}+\frac{2 \theta_{t 1} \theta_{t 2}(1-T)}{P T}+\frac{\theta_{t 2}\left(1-\theta_{t 2}\right)}{P^{2}}\right] \\
& =\frac{T^{2}}{n \pi^{2}}\left[\frac{P^{2} \theta_{t 1}(1-T)^{2}+T^{2} \theta_{t 2}}{P^{2} T^{2}}-\frac{\left[\theta_{t 1} P(1-T)-\theta_{t 2} T\right]^{2}}{P^{2} T^{2}}\right] \\
& =\frac{1}{n}\left[\frac{T(1-T)}{\pi}+\frac{T^{2}(1-P)}{\pi^{2} P}[1+\pi(1-T)]\right]
\end{aligned}
$$




$$
=\frac{T(1-T)}{n \pi}+\frac{(1-P) T^{2}(1+\pi-\pi T)}{n P \pi^{2}}
$$

Hence the theorem.

\section{HRRT2}

In this proposed method, a simple random sample of size $n$ is drawn with replacement from a finite population. The sampled individuals are required to reply a direct query as to whether or not they belong to A. When answering "No", the respondent is provided with a randomization device consisting of the statement "I belong to the sensitive group" with known probability $(1-P)$, exactly the same probability as used by Warner (1965), and the statement "Yes" with probability $P$ (Singh 1993, p. 68). The interviewee is instructed to use the device and report "Yes" or "No" for the random outcome of the sensitive statement according to his/her actual status. Otherwise, they are simply to report the "Yes" statement observed on the randomized response device. The whole procedure is completed by the respondent, unobserved by the interviewer. The probability of a "Yes" answer in the population is then

$$
\theta_{t 1}=\pi T
$$

and in the randomized response procedure is

$$
\theta_{t 3}=\pi(1-P)(1-T)+P
$$

The proposed estimators of $\pi$ and $T$ are given by

$$
\hat{\pi}_{a 2}=\frac{\left[(1-P) \hat{\theta}_{t 1}+\hat{\theta}_{t 3}-P\right]}{(1-P)}
$$

and

$$
\hat{T}_{2}=\frac{(1-P) \hat{\theta}_{t 1}}{\left[(1-P) \hat{\theta}_{t 1}+\hat{\theta}_{t 3}-p\right]},
$$




\section{TARRAY \& SINGH}

where $\hat{\theta}_{t 1}$ and $\hat{\theta}_{t 3}$ are the observed proportion of "Yes" answers. The principal properties of the estimator $\hat{\pi}_{a 2}$ are outlined in the following theorem:

Theorem 5. The estimator $\hat{\pi}_{a 2}$ is unbiased with variance given by

$$
\mathrm{V}\left(\hat{\pi}_{a 2}\right)=\frac{\pi(1-\pi)}{n}+\frac{P(1-\pi-\pi T)}{n(1-P)}
$$

Proof. The unbiasedness follows from $\mathrm{E}\left(\hat{\theta}_{t 1}\right)=\theta_{t 1}$ and $\mathrm{E}\left(\hat{\theta}_{t 3}\right)=\theta_{t 3}$. The variance of the estimator $\hat{\pi}_{a 2}$ can be obtained as follows:

$$
\begin{aligned}
\mathrm{V}\left(\hat{\pi}_{a 2}\right) & =\frac{1}{(1-P)^{2}}\left[(1-P)^{2} \mathrm{~V}\left(\hat{\theta}_{t 1}\right)+\mathrm{V}\left(\hat{\theta}_{t 3}\right)+2(1-P) \operatorname{cov}\left(\hat{\theta}_{t 1}, \hat{\theta}_{t 3}\right)\right] \\
& =\frac{1}{n(1-P)^{2}}\left[\left[(1-P)^{2} \theta_{t 1}+\theta_{t 3}\right]-\left[(1-P) \theta_{t 1}+\theta_{t 3}\right]^{2}\right] \\
& =\frac{1}{n(1-P)^{2}}\left[(1-P)^{2} \pi(1-\pi)+P(1-P)(1-\pi-\pi T)\right] \\
& =\frac{1}{2}\left[\pi(1-\pi)+\frac{P(1-\pi-\pi T)}{(1-P)}\right]
\end{aligned}
$$

Hence the theorem.

An unbiased estimator of the variance $\mathrm{V}\left(\hat{\pi}_{a 2}\right)$ can easily be obtained, which is given in the following theorem.

Theorem 6. The unbiased estimator of $\mathrm{V}\left(\hat{\pi}_{a 2}\right)$ is given by

$$
\mathrm{V}\left(\hat{\pi}_{a 2}\right)=\frac{\hat{\pi}_{a 2}\left(1-\hat{\pi}_{a 2}\right)}{n-1}+\frac{\left(1-\hat{\pi}_{a 2}-\hat{\theta}_{t 1}\right) P}{(n-1)(1-P)}
$$

To form an idea about the sampling fluctuation of the direct estimator $\hat{\pi}_{D 2}$ from the sample itself, one has to develop an estimator of $\operatorname{MSE}\left(\hat{\pi}_{a 2}\right)$. In fact, with 
the help of the proposed procedure, one can find an unbiased estimator of the MSE of $\hat{\pi}_{D 2}$, which is presented in the following theorem.

Theorem 7. The unbiased estimator of MSE of $\hat{\pi}_{D 2}$ is given by

$$
\begin{aligned}
\operatorname{MSE}\left(\hat{\pi}_{D}\right) & =\left(\hat{\theta}_{t 1}-\hat{\pi}_{a 2}\right)+\frac{2 \hat{\theta}_{t 1}}{(n-1)}\left[1-\hat{\pi}_{a 2}-\frac{P}{(1-P)}\right]-\hat{\mathrm{V}}\left(\hat{\pi}_{a 2}\right) \\
& =\left(\hat{\theta}_{t 1}-\hat{\pi}_{a 2}\right)^{2}+\frac{2 \hat{\theta}_{t 1}}{(n-1)(1-P)}\left[(1-P)-(1-P) \hat{\theta}_{t 1}-\hat{\theta}_{t 3}\right]-\hat{\mathrm{V}}\left(\hat{\pi}_{a 2}\right)
\end{aligned}
$$

Proof. The proof is straightforward and omitted.

Now to obtain the MSE of $\hat{T}_{2}$, we define $d_{1}^{*}=(1-P) \hat{\theta}_{t 1}$ and $d_{2}^{*}=\left[(1-P) \hat{\theta}_{t 1}+\hat{\theta}_{t 3}-P\right] \quad$. It follows that $\mathrm{E}\left(d_{1}^{*}\right)=(1-P) \pi T$ and $\mathrm{E}\left(d_{2}^{*}\right)=\left[(1-P) \theta_{t 1}+\theta_{t 3}-P\right]$. The estimator $\hat{T}_{2}$ can then be represented as $\hat{T}_{2}=d_{1}^{*} / d_{2}^{*}$, and we therefore have $T=\mathrm{E}\left(d_{1}^{*}\right) / \mathrm{E}\left(d_{2}^{*}\right)$. We then define the following quantities:

$$
e_{1}^{*}=\frac{d_{1}^{*}-\mathrm{E}\left(d_{1}^{*}\right)}{\mathrm{E}\left(d_{1}^{*}\right)}
$$

and

$$
e_{2}^{*}=\frac{d_{2}^{*}-\mathrm{E}\left(d_{2}^{*}\right)}{\mathrm{E}\left(d_{2}^{*}\right)},
$$

assuming that $\left|e_{1}\right|<1$ so that the function $\left(1+e_{2}\right)^{-1}$ can be validly expanded as a power series. It can be verified that 


\section{TARRAY \& SINGH}

$$
\begin{aligned}
\mathrm{E}\left(e_{1}^{* 2}\right) & =\frac{\theta_{t 1}\left(1-\theta_{t 1}\right)}{n \theta_{t 1}^{2}}=\frac{\theta_{t 1}\left(1-\theta_{t 1}\right)}{n \pi^{2} T^{2}} \\
\mathrm{E}\left(e_{2}^{* 2}\right) & =\frac{(1-P)^{2} \theta_{t 1}\left(1-\theta_{t 1}\right)+\theta_{t 3}\left(1-\theta_{t 3}\right)-2(1-P) \theta_{t 1} \theta_{t 3}}{n \pi^{2}(1-P)^{2}} \\
\mathrm{E}\left(e_{1}^{*} e_{2}^{*}\right) & =\frac{(1-P) \theta_{t 1}\left(1-\theta_{t 1}\right)-\theta_{t 1} \theta_{t 3}}{n \pi^{2} T(1-P)}
\end{aligned}
$$

and the estimation error of the estimator $\hat{T}_{2}$ can be expressed as

$$
\begin{aligned}
\hat{T}_{2}-T & =\frac{(1-P) \theta_{t 1}\left(1-e_{1}^{*}\right)-\theta_{t 1} \theta_{t 3}}{(1-P) \pi\left(1+e_{2}^{*}\right)}-T \\
& =\frac{(1-P) \pi T}{(1-P) \pi}\left(1-e_{1}^{*}\right)\left(1+e_{2}^{*}\right)^{-1}-T \\
& =T\left(1-e_{1}^{*}\right)\left(1+e_{2}^{*}\right)^{-1}-T \\
\hat{T}_{2}-T & =T\left(e_{1}^{*}-e_{2}^{*}\right)+\mathrm{o}_{P}\left(n^{-1 / 2}\right)
\end{aligned}
$$

Theorem 8. The MSE of the estimator $\hat{T}_{2}$, up to terms of order $\mathrm{o}\left(\mathrm{n}^{-1}\right)$, is given by

$$
\operatorname{MSE}\left(\hat{T}_{2}\right)=\frac{T(1-T)}{n \pi}+\frac{P T^{2}(1+\pi-\pi T)}{n(1-P) \pi^{2}}
$$

Proof.

$$
\begin{aligned}
\operatorname{MSE}\left(\hat{T}_{2}\right) & =\mathrm{E}\left(\hat{T}_{2}-T\right)^{2} \\
& \cong T^{2} \mathrm{E}\left(e_{1}^{*}-e_{2}^{*}\right)^{2} \\
& =T^{2}\left[\mathrm{E}\left(e_{1}^{* 2}\right)-2 \mathrm{E}\left(e_{1}^{*} e_{2}^{*}\right)+\mathrm{E}\left(e_{2}^{* 2}\right)\right]
\end{aligned}
$$




$$
\begin{aligned}
= & \frac{T^{2}}{n}\left[\begin{array}{c}
\frac{\theta_{t 1}\left(1-\theta_{t 1}\right)}{\pi^{2} T^{2}}-\frac{2\left[(1-P) \theta_{t 1}\left(1-\theta_{t 1}\right)-\theta_{t 1} \theta_{t 3}\right]}{(1-P) \pi^{2} T} \\
\left.+\frac{(1-P)^{2} \theta_{t 1}\left(1-\theta_{t 1}\right)+\theta_{t 3}\left(1-\theta_{t 3}\right)-2(1-P) \theta_{t 1} \theta_{t 3}}{(1-P)^{2} \pi^{2}}\right]
\end{array}\right] \\
= & \frac{T^{2}}{n \pi^{2}}\left[\begin{array}{c}
\left.\frac{\theta_{t 1}(1-T)^{2}}{T^{2}}+\frac{\theta_{t 3}}{(1-P)^{2}}-\left(\frac{\theta_{t 1}(1-T)}{T}-\frac{\theta_{t 3}}{(1-P)}\right)^{2}\right]
\end{array}\right] \\
= & \frac{1}{n}\left[\frac{T(1-T)}{\pi}+\frac{T^{2}(1-T)}{\pi(1-P)}+\frac{T^{2} P}{\pi^{2}(1-P)}\right] \\
= & \frac{1}{n}\left[\frac{T(1-T)}{\pi}+\frac{T^{2}(1-T)}{\pi(1-P)}-\frac{T(1-T)}{\pi}+\frac{T(1-T)^{2}}{\pi}+\frac{P T^{2}}{\pi^{2}(1-P)}\right] \\
= & \frac{1}{n}\left[\frac{T(1-T)}{\pi}-\frac{T^{2}(1-T)}{\pi}+\frac{(1-T) T^{2}}{\pi(1-P)}+\frac{P T^{2}}{\pi^{2}(1-P)}\right] \\
= & \frac{1}{n}\left[\frac{T(1-T)}{\pi}+\frac{T^{2}(1-T) P}{\pi(1-P)}+\frac{P T^{2}}{\pi^{2}(1-P)}\right] \\
= & \frac{1}{n}\left[\frac{T(1-T)}{\pi}+\frac{P T^{2}(1+\pi-\pi T)}{\pi^{2}(1-P)}\right]
\end{aligned}
$$

Hence the theorem.

\section{Theoretical Comparisons}

Comparisons of the proposed estimators $\hat{\pi}_{a 1}$ and $\hat{\pi}_{a 2}$ with Warner's estimator $\hat{\pi}_{W}$

From (3) and (8),

$$
\begin{aligned}
\mathrm{V}\left(\hat{\pi}_{W}\right)-\mathrm{V}\left(\hat{\pi}_{a 1}\right) & =\frac{(1-P)}{n}\left[\frac{P}{(2 P-1)^{2}}-\frac{1}{P}+\frac{\pi(1+t)}{P}\right] \\
& =\frac{(1-P)}{n(2 P-1)^{2} P}\left[P^{2}-(2 P-1)^{2}+\pi(1+T)(2 P-1)^{2}\right]
\end{aligned}
$$




\section{TARRAY \& SINGH}

which is positive if

$$
\left[(3 P-1)(1-P)+\pi(1+T)(2 P-1)^{2}\right]>0 \text {. }
$$

The condition (14) is always true as long as $P>1 / 3$. Thus the proposed estimator is more efficient than the Warner's (1965) estimator $\hat{\pi}_{W}$ if $P>1 / 3$.

It is further observed from (3) and (11) that $\mathrm{V}\left(\hat{\pi}_{W}\right)-\mathrm{V}\left(\hat{\pi}_{a 2}\right)>0$ if

$$
\frac{P}{n(2 P-1)^{2}(1-P)}\left[(1-P)^{2}-(2 P-1)^{2}(1-\pi-\pi T)\right]>0
$$

i.e. if $\left[P(2-3 P)+(2 P-1)^{2} \pi(1+T)\right]>0$, which is always true if $P<2 / 3$. Thus the proposed estimator $\hat{\pi}_{a 2}$ is better than Warner's (1965) estimator as long as $P<2 / 3$.

Comparisons of the proposed estimators $\left(\hat{\pi}_{a 1}, \hat{T}_{1}\right)$ with Huang's estimator $\left(\hat{\pi}_{H}, \hat{T}_{H}\right)$

From (7) and (8),

$$
\begin{aligned}
\mathrm{V}\left(\hat{\pi}_{H}\right)-\mathrm{V}\left(\hat{\pi}_{a 1}\right) & =\frac{(1-P)}{n}\left[\frac{(1-\pi T)}{(2 P-1)^{2} P}\left[P^{2}-(2 P-1)^{2}\right]+\frac{\pi}{P}\right] \\
& =\frac{(1-P)}{n P(2 P-1)^{2}}\left[(1-\pi T)(3 P-1)(1-P)+\pi(2 P-1)^{2}\right] \\
& =\frac{(1-P)}{n P(2 P-1)^{2}}\left[\begin{array}{c}
(3 P-1)(1-P) \\
-\pi\left[T(3 P-1)(1-P)-(2 P-1)^{2}\right]
\end{array}\right]
\end{aligned}
$$

which is positive if

$$
\left[(3 P-1)(1-P)-\pi\left(T(3 P-1)(1-P)-(2 P-1)^{2}\right)\right]>0
$$


i.e. if either

$$
\begin{aligned}
& \pi<\frac{(3 P-1)(1-P)}{\left[(3 P-1)(1-P) T-(2 P-1)^{2}\right]}, \quad P>\frac{1}{3} \text { and } \\
& T>\frac{(2 P-1)^{2}}{(3 P-1)(1-P)}
\end{aligned}
$$

or

$$
\begin{aligned}
& \pi>\frac{(3 P-1)(1-P)}{\left[(3 P-1)(1-P) T-(2 P-1)^{2}\right]}, \quad P<\frac{1}{3} \text { and } \\
& T>\frac{(2 P-1)^{2}}{(3 P-1)(1-P)}
\end{aligned}
$$

Thus the proposed estimator $\hat{\pi}_{a 1}$ is more efficient than Huang's (2004) estimator $\hat{\pi}_{H}$ as long as either inequality (16) or (17) is satisfied.

We note from (15) that the difference $\mathrm{V}\left(\hat{\pi}_{H}\right)-\mathrm{V}\left(\hat{\pi}_{a 1}\right)$ is always positive if

$$
\begin{aligned}
P^{2}-(2 P-1)^{2} & >0 \\
(3 P-1)(1-P) & >0
\end{aligned}
$$

i.e. if

$$
P>\frac{1}{3}
$$

which is a sufficient condition for the proposed estimator $\hat{\pi}_{a 1}$ to be more efficient than Huang's (2004) estimator $\hat{\pi}_{H}$.

From (7) and (11) we have 


\section{TARRAY \& SINGH}

$$
\begin{aligned}
\operatorname{MSE}\left(\hat{\pi}_{H}\right)-\operatorname{MSE}\left(\hat{T}_{1}\right) & =\frac{P(1-P) T^{2}(1-\pi T)}{n(2 P-1)^{2} \pi^{2}}-\frac{(1-P)(1+\pi-\pi T) T^{2}}{n P \pi^{2}} \\
& =\frac{(1-P) T^{2}}{n \pi^{2}}\left[\frac{P(1-\pi T)}{(2 P-1)^{2}}-\frac{(1+\pi-\pi T)}{P}\right] \\
& =\frac{(1-P) T^{2}}{n(2 P-1)^{2} \pi^{2}}\left[P^{2}(1-\pi T)-(2 P-1)^{2}(1+\pi-\pi T)\right]
\end{aligned}
$$

which is positive if

$$
\begin{array}{r}
{\left[P^{2}(1-\pi T)-(2 P-1)^{2}(1+\pi-\pi T)\right]>0} \\
{\left[P^{2}-(2 P-1)^{2}-\pi\left[T P^{2}+(1-T)(2 P-1)^{2}\right]\right]>0} \\
{\left[P^{2}-(2 P-1)^{2}-\pi\left[T\left(P^{2}-(2 P-1)^{2}\right)+(2 P-1)^{2}\right]\right]>0} \\
{\left[(3 P-1)(1-P)-\pi\left[T(3 P-1)(1-P)+(2 P-1)^{2}\right]\right]>0}
\end{array}
$$

i.e. if either

$$
\pi<\frac{(3 P-1)(1-P)}{\left[(2 P-1)^{2}+(3 P-1)(1-P) T\right]}, \quad P>\frac{1}{3}
$$

or

$$
\pi>\frac{(3 P-1)(1-P)}{\left[(2 P-1)^{2}+(3 P-1)(1-P) T\right]}, \quad P<\frac{1}{3}
$$

It follows that the proposed estimator $\hat{T}_{1}$ is better than Huang's (2004) estimator $\hat{T}_{H}$ if either (19) or (20) holds. 
Comparisons of the proposed estimators $\hat{\pi}_{a 2}$ and $\hat{T}_{2}$ with Huang's estimator $\left(\hat{\pi}_{H}, \hat{T}_{H}\right)$

From (6) and (11),

$$
\begin{aligned}
\mathrm{V}\left(\hat{\pi}_{H}\right)-\mathrm{V}\left(\hat{\pi}_{a 2}\right) & =\frac{P(1-P)}{n(2 P-1)^{2}}-\frac{P(1-\pi-\pi T)}{n(1-P)} \\
& =\frac{P}{n(1-P)(2 P-1)^{2}}\left[\pi\left(T P(3 P-2)+(2 P-1)^{2}\right)-P(3 P-2)\right]
\end{aligned}
$$

Which is positive if either

$$
\pi>\frac{P(3 P-2)}{\left[(2 P-1)^{2}+P(3 P-2) T\right]}, \quad P>\frac{2}{3}
$$

or

$$
\pi<\frac{P(3 P-2)}{\left[(2 P-1)^{2}+P(3 P-2) T\right]}, \quad P<\frac{2}{3} .
$$

Thus the proposed estimator $\hat{\pi}_{a 2}$ is better than Huang's (2004) estimator $\hat{T}_{H}$ if either (21) or (22) holds.

From (18) it is further observed that the different $\mathrm{V}\left(\hat{\pi}_{H}\right)-\mathrm{V}\left(\hat{\pi}_{a 2}\right)$ is always positive if $2-3 P>0$, i.e. if

$$
P<\frac{2}{3} \text {. }
$$

The condition (23) is sufficient for the proposed estimator $\hat{\pi}_{a 2}$ to be better than Huang's (2004) estimator $\hat{T}_{H}$.

From (7) and (12), 


$$
\begin{aligned}
\operatorname{MSE}\left(\hat{T}_{H}\right)-\mathrm{V}\left(\hat{T}_{2}\right) & =\frac{P T^{2}}{n \pi^{2}}\left[\frac{(1-P)(1-\pi T)}{(2 P-1)^{2}}-\frac{(1+\pi-\pi T)}{(1-P)}\right] \\
& =\frac{P T^{2}}{n \pi^{2}(1-P)(2 P-1)^{2}}\left[P(2-3 P)-\pi\left[P(2-3 P) T+(2 P-1)^{2}\right]\right]
\end{aligned}
$$

which is positive if either

$$
\pi<\frac{P(2-3 P)}{\left[P(2-3 P) T+P(2 P-1)^{2}\right]}, \quad P<\frac{2}{3}
$$

or

$$
\pi>\frac{P(2-3 P)}{\left[P(2-3 P) T+P(2 P-1)^{2}\right]}, \quad P>\frac{2}{3} .
$$

It follows that the proposed estimator $\hat{T}_{2}$ is more efficient than Huang's (2004) estimator $\hat{T}_{H}$ if either (24) or (25) is satisfied.

Comparisons of the proposed estimators $\left(\hat{\pi}_{a 2}, \hat{T}_{1}\right)$ with the proposed estimators $\left(\hat{\pi}_{a 2}, \hat{T}_{2}\right)$

From (7) and (11),

$$
\mathrm{V}\left(\hat{\pi}_{a 2}\right)-\mathrm{V}\left(\hat{\pi}_{a 1}\right)=\frac{(1-\pi-\pi T)(2 P-1)}{n P(1-P)}
$$

which is positive if either

$$
\pi<\frac{1}{1+T}, \quad P>\frac{1}{2}
$$

or 


$$
\pi<\frac{1}{1+T}, \quad P<\frac{1}{2}
$$

Thus the proposed estimator $\hat{\pi}_{a 1}$ is more efficient than the proposed estimator $\hat{\pi}_{a 2}$ if either (26) or (27) is satisfied.

Further, the difference $\mathrm{V}\left(\hat{\pi}_{a 2}\right)-\mathrm{V}\left(\hat{\pi}_{a 1}\right)<0$ if

$$
\frac{(1-\pi-\pi T)(2 P-1)}{n P(1-P)}<0
$$

i.e. if $[1-\pi(1+T)](2 P-1)<0$,

$$
\pi>\frac{1}{1+T}, \quad P>\frac{1}{2}
$$

or

$$
\pi<\frac{1}{1+T}, \quad P<\frac{1}{2}
$$

It follows from the above that the proposed estimator $\hat{\pi}_{a 2}$ is more efficient than the suggested estimator $\hat{\pi}_{a 1}$ if either (28) or (29) is satisfied.

From (10) and (12) we have

$$
\operatorname{MSE}\left(\hat{T}_{2}\right)-\operatorname{MSE}\left(\hat{T}_{1}\right)=\frac{T^{2}}{n \pi^{2}}(1+\pi-\pi T)\left[\frac{P}{1-P}-\frac{(1-P)}{P}\right],
$$

which is positive if $P^{2}-(1-P)^{2}>0$, i.e. if

$$
P>\frac{1}{2}
$$

Thus if $P>1 / 2$ holds, the proposed estimator $\hat{T}_{1}$ is better than the estimator $\hat{T}_{2}$. On the other hand, for $P<1 / 2$, the proposed estimator $\hat{T}_{2}$ would be better than $\hat{T}_{1}$. 
TARRAY \& SINGH

Comparisons of the proposed estimators $\left(\hat{\pi}_{a 1}, \hat{\pi}_{a 2}\right)$ with direct estimator $\hat{\pi}_{D}$.

From (2) and (7) we have

$$
\mathrm{V}\left(\hat{\pi}_{D}\right)-\mathrm{V}\left(\hat{\pi}_{a 1}\right)=\frac{1}{n P}\left[\begin{array}{c}
n P \pi^{2}(1-T)^{2}+\pi T(1-\pi T) P \\
-P \pi(1-\pi)-(1-P)(1-\pi-\pi T)
\end{array}\right]
$$

which is greater than zero if

$$
\left[n P \pi^{2}\left(1-T^{2}\right)+\pi T(1-\pi T) P-P \pi(1-\pi)-(1-P)(1-\pi-\pi T)\right]>0
$$

i.e. if

$$
n>\frac{1}{P \pi^{2}\left(1-T^{2}\right)}[P \pi(1-T)(1-\pi-\pi T)+(1-P)(1-\pi-\pi T)]
$$

i.e. if

$$
n>\frac{(1-\pi-\pi T)}{P \pi^{2}(1-T)^{2}}[P \pi(1-T)+(1-P)], \quad \pi<\frac{1}{(1+T)} .
$$

Thus the proposed estimator $\hat{\pi}_{a 1}$ is more efficient than the direct estimator $\hat{\pi}_{D}$ if the inequality (31) holds.

From (2) and (11) we have

$$
\operatorname{MSE}\left(\hat{\pi}_{D}\right)-\operatorname{MSE}\left(\hat{\pi}_{a 2}\right)=\frac{1}{n(1-P)}\left[\begin{array}{c}
n(1-P) \pi^{2}(1-T)^{2}+\pi T(1-\pi T)(1-P) \\
-(1-P) \pi(1-\pi)-P(1-\pi-\pi T)
\end{array}\right],
$$

which is greater than zero if

$$
n(1-P) \pi^{2}(1-T)^{2}+\pi T(1-\pi T)(1-P)-(1-P) \pi(1-\pi)-P(1-\pi-\pi T)>0
$$

i.e. if 


$$
\begin{aligned}
& n>\frac{1}{(1-P) \pi^{2}(1-T)^{2}}[(1-P) \pi(1-\pi)+P(1-\pi-\pi T)-(1-P) \pi T(1-\pi T)] \\
& n>\frac{1}{(1-P) \pi^{2}(1-T)^{2}}[(1-P) \pi(1-T)(1-\pi-\pi T)+P(1-\pi-\pi T)] \\
& n>\frac{(1-\pi-\pi T)}{(1-P) \pi^{2}(1-T)^{2}}[(1-P) \pi(1-T)+P]
\end{aligned}
$$

i.e. if

$$
\pi<\frac{1}{(1+T)} .
$$

It follows that the proposed estimator $\hat{\pi}_{a 2}$ is more efficient than the direct estimator $\hat{\pi}_{D}$ if the condition (32) holds.

\section{Numerical Illustration}

This illustration is provided to give a tangible idea about the magnitude of the relative efficiency of the suggested procedures with respect to the Huang (2004) and direct estimator procedures. The percent relative efficiency (PRE) of the proposed estimators $\left(\hat{\pi}_{a 1}, \hat{\pi}_{a 2}\right)$ in relation to the Huang (2004) estimator $\hat{\pi}_{H}$ are given by

$$
\operatorname{PRE}\left(\hat{\pi}_{a 1}, \hat{\pi}_{H}\right)=\frac{P\left[(2 P-1)^{2} \pi(1-\pi)+P(1-P)(1-\pi T)\right]}{(2 P-1)^{2}[P \pi(1-\pi)+(1-P)(1-\pi-\pi T)]} \times 100
$$

and

$$
\operatorname{PRE}\left(\hat{\pi}_{a 2}, \hat{\pi}_{H}\right)=\frac{(1-P)\left[(2 P-1)^{2} \pi(1-\pi)+P(1-P)(1-\pi T)\right]}{(2 P-1)^{2}[(1-P) \pi(1-\pi)+P(1-\pi-\pi T)]} \times 100,
$$




\section{TARRAY \& SINGH}

respectively. The PRE of the proposed estimators $\left(\hat{T}_{1}, \hat{T}_{2}\right)$ with respect to the Huang (2004) estimator $\hat{T}_{H}$ are given by

$$
\operatorname{PRE}\left(\hat{T}_{1}, \hat{T}_{H}\right)=\frac{P\left[(1-T)(2 P-1)^{2} \pi+P(1-P) T(1-\pi T)\right]}{(2 P-1)^{2}[(1-T) P \pi+(1-P) T(1+\pi-\pi T)]} \times 100
$$

and

$$
\operatorname{PRE}\left(\hat{T}_{2}, \hat{T}_{H}\right)=\frac{(1-P)\left[(1-T)(2 P-1)^{2} \pi+T P(1-P)(1-\pi T)\right]}{(2 P-1)^{2}[(1-T)(1-P) \pi+P T(1+\pi-\pi T)]} \times 100
$$

respectively. The expression for PRE of the proposed estimators $\left(\hat{\pi}_{a 1}, \hat{\pi}_{a 2}\right)$ in relation to the direct estimator $\hat{\pi}_{D}$ are given by

$$
\operatorname{PRE}\left(\hat{\pi}_{a 1}, \hat{\pi}_{D}\right)=\frac{P\left[\left(\pi T(1-\pi T)+n \pi^{2}(1-T)^{2}\right)\right]}{P \pi(1-\pi)+(1-P)(1-\pi-\pi T)} \times 100
$$

and

$$
\operatorname{PRE}\left(\hat{\pi}_{a 2}, \hat{\pi}_{D}\right)=\frac{(1-P)\left[\left(\pi T(1-\pi T)+n \pi^{2}(1-T)^{2}\right)\right]}{(1-P) \pi(1-\pi)+P(1-\pi-\pi T)} \times 100
$$

respectively. For comparing the two proposed procedures, we give the PRE of $\left(\hat{\pi}_{a 1}, \hat{T}_{1}\right)$ with respect to $\left(\hat{\pi}_{a 2}, \hat{T}_{2}\right)$ as

$$
\operatorname{PRE}\left(\hat{\pi}_{a 1}, \hat{\pi}_{a 2}\right)=\frac{P[(1-P) \pi(1-\pi)+P(1-\pi-\pi T)]}{[P \pi(1-\pi)+(1-P)(1-\pi-\pi T)](1-P)} \times 100
$$

and 


$$
\operatorname{PRE}\left(\hat{T}_{1}, \hat{T}_{2}\right)=\frac{P\left[(1-P) \pi T(1-T)+P T^{2}(1+\pi-\pi T)\right]}{\left[P \pi T(1-T)+(1-P) T^{2}(1+\pi-\pi T)\right](1-P)} \times 100,
$$

respectively. We have further obtained the expressions for PRE of the proposed estimators $\left(\hat{\pi}_{a 2}, \hat{T}_{2}\right)$ with respect to $\left(\hat{\pi}_{a 1}, \hat{T}_{1}\right)$, given by

$$
\operatorname{PRE}\left(\hat{\pi}_{a 2}, \hat{\pi}_{a 1}\right)=\frac{(1-P)[P \pi(1-\pi)+(1-P)(1-\pi-\pi T)]}{P[(1-P) \pi(1-\pi)+P(1-\pi-\pi T)]} \times 100
$$

and

$$
\operatorname{PRE}\left(\hat{T}_{2}, \hat{T}_{1}\right)=\frac{(1-P)\left[P \pi T(1-T)+(1-P) T^{2}(1+\pi-\pi T)\right]}{P\left[(1-P) \pi T(1-T)+P T^{2}(1+\pi-\pi T)\right]} \times 100,
$$

Respectively.

Using the formulae (33)-(42), we have computed

(i) $\quad \operatorname{The} \operatorname{PRE}\left(\hat{\pi}_{a 1}, \hat{\pi}_{H}\right)$ and $\operatorname{PRE}\left(\hat{T}_{1}, \hat{T}_{H}\right)$ for the values of $P=0.6,0.7$, $0.8 ; T=0.10,0.15,0.30,0.45,0.60 ;$ and $\pi=0.1$ (0.1) 0.9. The results are displayed in Tables 1 and 3 .

(ii) The $\operatorname{PRE}\left(\hat{\pi}_{a 2}, \hat{\pi}_{H}\right)$ and $\operatorname{PRE}\left(\hat{T}_{2}, \hat{T}_{H}\right)$ for the values of $P=0.20$, $0.30,0.40 ; T=0.10,0.15,0.30,0.45,0.60$; and $\pi=0.1(0.1) 0.9$. The results are shown in Tables 2 and 4.

(iii) $\quad \operatorname{The} \operatorname{PRE}\left(\hat{\pi}_{a 1}, \hat{\pi}_{D}\right)$ for the values of $P=0.60,0.70,0.80 ; T=0.10$, $0.15,0.30,0.45,0.60 ; \pi=0.1(0.1) 0.9$; and $n=1000$. The findings are displayed in Table 5 .

(iv) The $\operatorname{PRE}\left(\hat{\pi}_{a 2}, \hat{T}_{D}\right)$ for the values of $P=0.2,0.30,0.40 ; T=0.10$, $0.15,0.30,0.45,0.60 ; \pi=0.1(0.1) 0.9$; and $n=1000$. The findings are displayed in Table 6.

(v) The $\operatorname{PRE}\left(\hat{\pi}_{a 1}, \hat{\pi}_{a 2}\right)$ and $\operatorname{PRE}\left(\hat{T}_{1}, \hat{T}_{2}\right)$ for the values of $P=0.6,0.7$, $0.8 ; T=0.10,0.15,0.30,0.45,0.60 ;$ and $\pi=0.1(0.1) 0.9$. The findings are displayed in Tables 7 and 8 . 


\section{TARRAY \& SINGH}

(vi) The $\operatorname{PRE}\left(\hat{\pi}_{a 2}, \hat{\pi}_{a 1}\right)$ and $\operatorname{PRE}\left(\hat{T}_{2}, \hat{T}_{1}\right)$ for the values of $P=0.20,0.30$, $0.40 ; T=0.10,0.15,0.30,0.45,0.60$; and $\pi=0.1(0.1) 0.9$. The results are displayed in Tables 9 and 10.

Tables 1 and 3 show that the values of $\operatorname{PRE}\left(\hat{\pi}_{a 1}, \hat{\pi}_{H}\right)$ and $\operatorname{PRE}\left(\hat{T}_{1}, \hat{T}_{H}\right)$ are larger than 100 , showing that $\hat{\pi}_{H}$ is more efficient than $\hat{\pi}_{a 1}$ and that $\hat{T}_{1}$ is also superior to $\hat{T}_{H}$. It is observed that, for fixed values of $(P, T)$, the $\operatorname{PRE}\left(\hat{\pi}_{a 1}, \hat{\pi}_{H}\right)\left\lfloor\operatorname{PRE}\left(\hat{T}_{1}, \hat{T}_{H}\right)\right\rfloor$ decreases (increases) as $\pi$ increases (decreases) slowly (rapidly). For fixed values of $(T, \pi)$, both $\operatorname{PRE}\left(\hat{\pi}_{a 1}, \hat{\pi}_{H}\right)$ and $\operatorname{PRE}\left(\hat{T}_{1}, \hat{T}_{H}\right)$ decrease in a speedy manner as $P$ increases. Thus a larger gain in efficiency by using $\hat{\pi}_{a 1}\left(\hat{T}_{1}\right)$ over $\hat{\pi}_{H}\left(\hat{T}_{H}\right)$ is expected when $P$ is close to 0.5 .

Tables 2 and 4 exhibit that

- $\quad$ For fixed values of $(P, T)$, the $\operatorname{PRE}\left(\hat{\pi}_{a 2}, \hat{\pi}_{H}\right)\left\lfloor\operatorname{PRE}\left(\hat{T}_{2}, \hat{T}_{H}\right)\right\rfloor$ increases (decreases) as $\pi$ increases (decreases)

- $\quad \operatorname{PRE}\left(\hat{\pi}_{a 2}, \hat{\pi}_{H}\right)\left\lfloor\operatorname{PRE}\left(\hat{T}_{2}, \hat{T}_{H}\right)\right\rfloor$ decreases (increases) slowly (rapidly) as $T$ increases (decreases)

- $\quad$ Both $\operatorname{PRE}\left(\hat{\pi}_{a 2}, \hat{\pi}_{H}\right)$ and $\operatorname{PRE}\left(\hat{T}_{2}, \hat{T}_{H}\right)$ increase in a speedy way as $P$ increases

Hence higher gain in efficiency by using $\hat{\pi}_{a 2}\left(\hat{T}_{2}\right)$ is observed when $(P, \pi)$ are closer to 0.5. It is further observed from Tables 2 and 4 that the values of $\operatorname{PRE}\left(\hat{\pi}_{a 2}, \hat{\pi}_{H}\right)$ and $\operatorname{PRE}\left(\hat{T}_{2}, \hat{T}_{H}\right)$ are greater than 100 , from which it follows that the envisaged estimators $\left(\hat{\pi}_{a 2}, \hat{T}_{2}\right)$ are more efficient than Huang's (2004) estimators $\left(\hat{\pi}_{H}, \hat{T}_{H}\right)$.

It is observed from Table 5 that

- $\quad$ For fixed $(P, T)$, the $\operatorname{PRE}\left(\hat{\pi}_{a 1}, \hat{\pi}_{D}\right)$ increases as $\pi$ increases 
- $\quad$ For fixed $(P, \pi)$, the $\operatorname{PRE}\left(\hat{\pi}_{a 1}, \hat{\pi}_{D}\right)$ decreases as $T$ increases

- $\quad$ For fixed $(T, \pi)$, the $\operatorname{PRE}\left(\hat{\pi}_{a 1}, \hat{\pi}_{D}\right)$ increases as $P$ increases

There is substantial gain in efficiency through use of the proposed estimator $\hat{\pi}_{a 1}$ over direct estimator $\hat{\pi}_{D}$ for all values of $(P, \pi, T)$ considered here.

It is observed from Table 6 that

- $\quad$ For fixed $(P, T)$, the $\operatorname{PRE}\left(\hat{\pi}_{a 2}, \hat{\pi}_{D}\right)$ increases as $\pi$ increases

- $\quad$ For fixed $(\mathrm{P}, \pi)$, the $\operatorname{PRE}\left(\hat{\pi}_{a 2}, \hat{\pi}_{D}\right)$ decreases as $T$ increases

- $\quad$ For fixed $(T, \pi)$, the $\operatorname{PRE}\left(\hat{\pi}_{a 2}, \hat{\pi}_{D}\right)$ increases as $P$ increases

There is substantial gain in efficiency through use of the proposed estimator $\hat{\pi}_{a 2}$ over direct estimator $\hat{\pi}_{D}$ for all values of $(P, \pi, T)$ considered here.

Tables 5 and 6 clearly demonstrate the superiority of the proposed estimators $\hat{\pi}_{a 1}$ and $\hat{\pi}_{a 2}$ over the usual direct estimator $\hat{\pi}_{D}$ as the values of $\operatorname{PRE}\left(\hat{\pi}_{a 1}, \hat{\pi}_{D}\right)$ and $\operatorname{PRE}\left(\hat{\pi}_{a 2}, \hat{\pi}_{D}\right)$ are larger than 100 for all values of $(P, \pi, T)$ considered.

Tables 7 and 8 demonstrate that the values of $\operatorname{PRE}\left(\hat{\pi}_{a 1}, \hat{\pi}_{a 2}\right)$ and $\operatorname{PRE}\left(\hat{T}_{1}, \hat{T}_{2}\right)$ are greater than 100 for $0.10 \leq T \leq 0.60,0.10 \leq T \leq 0.50$, and $P>1 / 2$. Both $\operatorname{PRE}\left(\hat{\pi}_{a 1}, \hat{\pi}_{a 2}\right)$ and $\operatorname{PRE}\left(\hat{T}_{1}, \hat{T}_{2}\right)$ increase in a speedy way as $P$ increases. Hence higher gain in efficiency by using $\operatorname{PRE}\left(\hat{\pi}_{a 1}, \hat{\pi}_{a 2}\right)$ and $\operatorname{PRE}\left(\hat{T}_{1}, \hat{T}_{2}\right)$ is observed when $(P, \pi)$ are closer to 0.5 .

It is observed from Tables 9 and 10 that the values of $\operatorname{PRE}\left(\hat{\pi}_{a 2}, \hat{\pi}_{a 1}\right)$ and $\operatorname{PRE}\left(\hat{T}_{2}, \hat{T}_{1}\right)$ are greater than 100 for $0.10 \leq T \leq 0.60,0.10 \leq T \leq 0.50$, and $P<1 / 2$. Thus the proposed procedures $\left(\hat{\pi}_{a 2}, \hat{T}_{2}\right)$ are more efficient than the estimators $\left(\hat{\pi}_{a 1}, \hat{T}_{1}\right)$. Higher gains in efficiencies are observed for lower values of $P$ (i.e. for the values of $P$ close to zero).

Finally we conclude that the proposed procedures are superior to the Huang (2004) procedure and hence the Chang and Huang (2001) procedure, and to the usual direct procedure. 


\section{TARRAY \& SINGH}

\section{Conclusion}

Randomized response procedures are attractive mechanisms for counteracting fears in response and providing with valid statistical inferences concerning a population. The proposed randomized response procedure allows us to estimate the population proportion $\pi$ unbiasedly and to get an admissible estimator for $T$, which is an unattainable feature for most of the competing methods. It has been shown theoretically and empirically that the proposed procedures are better than the Warner (1965), Chang and Huang (2001), and Huang (2004) procedures. The unbiased estimators of the MSE are provided for the direct response survey based on the proposed RR techniques. The suggested procedure is therefore recommended for application in survey sampling practice.

\section{References}

Chang, H. J. \& Huang K. C. (2001). Estimation of proportion and sensitivity of a qualitative character. Metrika, 53(3), 269-280. doi: 10.1007/s001840100109

Chang, H. J., Wang, C. L., \& Huang, K. C. (2004a). On estimating the proportion of a qualitative sensitive character using randomized response sampling. Quality and Quantity, 38(5), 675-680. doi: 10.1007/s11135-005-8105-4

Chang, H. J., Wang, C. L., \& Huang, K. C. (2004b). Using randomized response to estimate the proportion and truthful reporting probability in a dichotomous finite population. Journal of Applied Statistics, 31(5), 565-573. doi: 10.1080/02664760410001681819

Chua, T. C. \& Tsui, A. K. (2000). Procuring honest responses indirectly. Journal of Statistics Planning and Inference, 90(1), 107-116. doi: 10.1016/S03783758(00)00109-9

Fox, J. A. \& Tracy, P. E. (1986). Randomized response: A method of sensitive surveys. Newbury Park, CA: SEGE Publications.

Huang, K. C. (2004). Survey technique for estimating the proportion and sensitivity in a dichotomous finite population. Statistica Neerlandica, 58(1), 7582. doi: 10.1046/j.0039-0402.2003.00113.x

Mahmood, M., Singh, S. \& Horn, S. (1998). On the confidentiality guaranteed under randomized response sampling: A comparison with several new techniques. Biomedical Journal, 40(2), 237-242. doi: 10.1002/(SICI)15214036(199806)40:2<237::AID-BIMJ237>3.0.CO;2-N 
Mangat, N. S. (1994). An improved randomized response strategy. Journal of the Royal Statistical Society. Series B, 56(1), 93-95.

Mangat, N. S. \& Singh, R. (1990). An alternative randomized procedure. Biometrika, 77(2), 439-442. doi: 10.1093/biomet/77.2.439

Sing, S., Singh, R., and Mangat, N. S. (2000). Some alternative strategies to Moor's model in randomized response model. Journal of Statistical Planning and Inference, 83(1), 243-255.

Singh, H. P. \& Tarray, T. A. (2012). A stratified unknown repeated trials in randomized response sampling. Commmunications for Statistical Applications and Methods, 19(6), 751-759. doi: 10.5351/CKSS.2012.19.6.751

Singh, H. P. \& Tarray, T. A. (2013a). A modified survey technique for estimating the proportion and sensitivity in a dichotomous finite population. International Journal of Advanced Scientific and Technical Research, 3(6), 459472.

Singh, H. P. \& Tarray, T. A. (2013b). An alternative to Kim and Warde's mixed randomized response model. Statistics and Operations Research Transactions, 37(2), 189-210.

Singh, H. P. \& Tarray, T. A. (2013c). An alternative to Kim and Warde's mixed randomized response technique. Statistica, 73(3), 379-402.

Singh, H. P. \& Tarray, T. A. (2014a). An alternative to stratified Kim and Warde's randomized response model using optimal (Neyman) allocation. Model Assisted Statistics and Applications, 9(1), 37-62.

Singh, H. P. \& Tarray, T. A. (2014b). An improved mixed randomized response model. Model Assisted Statistics and Applications, 9(1), 73-87.

Singh, H. P. \& Tarray, T. A. (2014c). A dexterous randomized response model for estimating a rare sensitive attribute using Poisson distribution. Statistics \& Probability Letters, 90, 42-45. doi: 10.1016/j.spl.2014.03.019

Singh, H. P. \& Tarray, T. A. (2014d). A modified mixed randomized response model. Statistics in Transition new series, 15(1), 67-82.

Singh, H. P. \& Tarray, T. A. (2014e). An improvement over Kim and Elam stratified unrelated question randomized response model using Neyman allocation. Sankhya B, 77(1). doi: 10.1007/s13571-014-0088-5

Singh, R. \& Mangat, N. S. (1996). Elements of survey sampling (Vol. 15). Dordrecht, Netherlands: Kluwer Academic Publishers.

Singh, S. (1993). An alternative to Warner's randomized response technique. Statistica, 53(1), 67-71. 


\section{TARRAY \& SINGH}

Warner, S. L. (1965). Randomized response: A survey technique for

eliminating evasive answer bias. Journal of the American Statistical Association, 60(309), 63-69. doi: 10.1080/01621459.1965.10480775 


\section{Appendix}

Table 1. The present relative efficiency of the proposed estimator $\hat{\pi}_{a 1}$ with respect to Huang (2004) estimator $\hat{\pi}_{H}$, i.e. $\operatorname{PRE}\left(\hat{\pi}_{a 1}, \hat{\pi}_{H}\right)$.

\begin{tabular}{|c|c|c|c|c|c|c|}
\hline \multirow[b]{2}{*}{$P$} & & \multicolumn{5}{|c|}{$\pi$} \\
\hline & $T$ & 0.1 & 0.2 & 0.3 & 0.4 & 0.5 \\
\hline 0.6 & 0.10 & 882.44 & 888.24 & 918.27 & 978.26 & 1081.82 \\
\hline 0.6 & 0.15 & 882.35 & 888.12 & 918.56 & 980.00 & 1087.50 \\
\hline 0.6 & 0.30 & 882.09 & 887.76 & 919.46 & 985.71 & 1106.90 \\
\hline 0.6 & 0.45 & 881.82 & 887.37 & 920.45 & 992.31 & 1130.77 \\
\hline 0.6 & 0.60 & 881.54 & 886.96 & 921.56 & 1000.00 & 1160.87 \\
\hline 0.7 & 0.10 & 294.72 & 292.59 & 298.33 & 312.50 & 338.00 \\
\hline 0.7 & 0.15 & 294.66 & 292.47 & 298.23 & 312.61 & 338.79 \\
\hline 0.7 & 0.30 & 294.50 & 292.10 & 297.90 & 312.98 & 341.41 \\
\hline 0.7 & 0.45 & 294.34 & 291.71 & 297.54 & 313.39 & 344.48 \\
\hline 0.7 & 0.60 & 294.17 & 291.30 & 297.15 & 313.86 & 348.14 \\
\hline 0.8 & 0.10 & 169.60 & 167.76 & 169.83 & 175.44 & 185.44 \\
\hline 0.8 & 0.15 & 169.57 & 167.69 & 169.75 & 175.41 & 185.58 \\
\hline 0.8 & 0.30 & 169.47 & 167.47 & 169.50 & 175.31 & 186.01 \\
\hline 0.8 & 0.45 & 169.36 & 167.24 & 169.24 & 175.20 & 186.49 \\
\hline 0.8 & 0.60 & 169.26 & 167.00 & 168.95 & 175.08 & 187.04 \\
\hline
\end{tabular}

Table 2. The present relative efficiency of the proposed estimator $\hat{\pi}_{a 2}$ with respect to Huang (2004) estimator $\hat{\pi}_{H}$, i.e. $\operatorname{PRE}\left(\hat{\pi}_{a 2}, \hat{\pi}_{H}\right)$.

\begin{tabular}{rrrrrrr} 
& \multicolumn{7}{c}{$\boldsymbol{\pi}$} \\
\cline { 3 - 7 } $\boldsymbol{P}$ & $\boldsymbol{T}$ & $\mathbf{0 . 1}$ & $\mathbf{0 . 2}$ & $\mathbf{0 . 3}$ & $\mathbf{0 . 4}$ & $\mathbf{0 . 5}$ \\
\hline 0.2 & 0.10 & 169.60 & 167.76 & 169.83 & 175.44 & 185.44 \\
0.2 & 0.15 & 169.57 & 167.69 & 169.75 & 175.41 & 185.58 \\
0.2 & 0.30 & 169.47 & 167.47 & 169.50 & 175.31 & 186.01 \\
0.2 & 0.45 & 169.36 & 167.24 & 169.24 & 175.20 & 186.49 \\
0.2 & 0.60 & 169.26 & 167.00 & 168.95 & 175.08 & 187.04 \\
0.3 & 0.10 & 294.72 & 292.59 & 298.33 & 312.50 & 338.00 \\
0.3 & 0.15 & 294.66 & 292.47 & 298.23 & 312.61 & 338.79 \\
0.3 & 0.30 & 294.50 & 292.10 & 297.90 & 312.98 & 341.41 \\
0.3 & 0.45 & 294.34 & 291.71 & 297.54 & 313.39 & 344.48 \\
0.3 & 0.60 & 294.17 & 291.30 & 297.15 & 313.86 & 348.14 \\
0.4 & 0.10 & 882.44 & 888.24 & 918.27 & 978.26 & 1081.82 \\
0.4 & 0.15 & 882.35 & 888.12 & 918.56 & 980.00 & 1087.50 \\
0.4 & 0.30 & 882.09 & 887.76 & 919.46 & 985.71 & 1106.90 \\
0.4 & 0.45 & 881.82 & 887.37 & 920.45 & 992.31 & 1130.77 \\
0.4 & 0.60 & 881.54 & 886.96 & 921.56 & 1000.00 & 1160.87 \\
\hline
\end{tabular}




\section{TARRAY \& SINGH}

Table 3. The present relative efficiency of the proposed estimator $\hat{T}_{1}$ with respect to Huang (2004) estimator $\hat{T}_{H}$, i.e. $\operatorname{PRE}\left(\hat{T}_{1}, \hat{T}_{H}\right)$.

\begin{tabular}{|c|c|c|c|c|c|c|}
\hline \multirow[b]{2}{*}{$P$} & & \multicolumn{5}{|c|}{$\pi$} \\
\hline & $T$ & 0.1 & 0.2 & 0.3 & 0.4 & 0.5 \\
\hline 0.6 & 0.10 & 420.49 & 296.91 & 240.23 & 207.69 & 186.59 \\
\hline 0.6 & 0.15 & 502.07 & 363.41 & 292.90 & 250.21 & 221.59 \\
\hline 0.6 & 0.30 & 639.44 & 497.83 & 408.85 & 347.76 & 303.23 \\
\hline 0.6 & 0.45 & 708.88 & 579.46 & 486.01 & 415.36 & 360.08 \\
\hline 0.6 & 0.60 & 750.88 & 634.38 & 540.85 & 464.10 & 400.00 \\
\hline 0.7 & 0.10 & 160.87 & 133.85 & 122.47 & 116.19 & 112.21 \\
\hline 0.7 & 0.15 & 180.24 & 147.21 & 131.98 & 123.21 & 117.52 \\
\hline 0.7 & 0.30 & 217.73 & 178.01 & 155.46 & 140.92 & 130.77 \\
\hline 0.7 & 0.45 & 239.51 & 199.79 & 173.45 & 154.70 & 140.67 \\
\hline 0.7 & 0.60 & 253.80 & 216.09 & 187.68 & 165.51 & 147.72 \\
\hline 0.8 & 0.10 & 114.29 & 106.71 & 103.77 & 102.20 & 101.23 \\
\hline 0.8 & 0.15 & 119.87 & 109.72 & 105.50 & 103.18 & 101.72 \\
\hline 0.8 & 0.30 & 132.67 & 117.66 & 110.17 & 105.67 & 102.68 \\
\hline 0.8 & 0.45 & 141.63 & 124.32 & 114.17 & 107.49 & 102.76 \\
\hline 0.8 & 0.60 & 148.30 & 130.03 & 117.59 & 108.58 & 101.75 \\
\hline
\end{tabular}

Table 4. The present relative efficiency of the proposed estimator $\hat{T}_{2}$ with respect to Huang (2004) estimator $\hat{T}_{H}$, i.e. $\operatorname{PRE}\left(\hat{T}_{2}, \hat{T}_{H}\right)$.

\begin{tabular}{|c|c|c|c|c|c|c|}
\hline \multirow[b]{2}{*}{$P$} & \multirow[b]{2}{*}{$T$} & \multicolumn{5}{|c|}{$\pi$} \\
\hline & & 0.1 & 0.2 & 0.3 & 0.4 & 0.5 \\
\hline 0.2 & 0.10 & 114.29 & 106.71 & 103.77 & 102.20 & 101.23 \\
\hline 0.2 & 0.15 & 119.87 & 109.72 & 105.50 & 103.18 & 101.72 \\
\hline 0.2 & 0.30 & 132.67 & 117.66 & 110.17 & 105.67 & 102.68 \\
\hline 0.2 & 0.45 & 141.63 & 124.32 & 114.17 & 107.49 & 102.76 \\
\hline 0.2 & 0.60 & 148.30 & 130.03 & 117.59 & 108.58 & 101.75 \\
\hline 0.3 & 0.10 & 160.87 & 133.85 & 122.47 & 116.19 & 112.21 \\
\hline 0.3 & 0.15 & 180.24 & 147.21 & 131.98 & 123.21 & 117.52 \\
\hline 0.3 & 0.30 & 217.73 & 178.01 & 155.46 & 140.92 & 130.77 \\
\hline 0.3 & 0.45 & 239.51 & 199.79 & 173.45 & 154.70 & 140.67 \\
\hline 0.3 & 0.60 & 253.80 & 216.09 & 187.68 & 165.51 & 147.72 \\
\hline 0.4 & 0.10 & 420.49 & 296.91 & 240.23 & 207.69 & 186.59 \\
\hline 0.4 & 0.15 & 502.07 & 363.41 & 292.90 & 250.21 & 221.59 \\
\hline 0.4 & 0.30 & 639.44 & 497.83 & 408.85 & 347.76 & 303.23 \\
\hline 0.4 & 0.45 & 708.88 & 579.46 & 486.01 & 415.36 & 360.08 \\
\hline 0.4 & 0.60 & 750.88 & 634.38 & 540.85 & 464.10 & 400.00 \\
\hline
\end{tabular}


Table 5. The present relative efficiency of the proposed estimator $\hat{\pi}_{a 1}$ with respect to the direct estimator $\hat{\pi}_{D}$, i.e. $\operatorname{PRE}\left(\hat{\pi}_{a 1}, \hat{\pi}_{D}\right)$.

\begin{tabular}{rrrrrrr} 
& \multicolumn{7}{c}{$\boldsymbol{\pi}$} \\
\cline { 3 - 7 } $\boldsymbol{P}$ & $\boldsymbol{T}$ & $\mathbf{0 . 1}$ & $\mathbf{0 . 2}$ & $\mathbf{0 . 3}$ & $\mathbf{0 . 4}$ & $\mathbf{0 . 5}$ \\
\hline 0.6 & 0.10 & 1186.81 & 4767.59 & 11105.95 & 21136.70 & 36826.82 \\
0.6 & 0.15 & 1064.67 & 4296.40 & 10062.06 & 19276.07 & 33880.20 \\
0.6 & 0.30 & 735.69 & 3008.63 & 7164.63 & 14018.86 & 25371.21 \\
0.6 & 0.45 & 464.84 & 1923.46 & 4660.53 & 9336.08 & 17492.16 \\
0.6 & 0.60 & 254.83 & 1060.70 & 2613.34 & 5371.33 & 10489.57 \\
0.7 & 0.10 & 1720.28 & 6558.88 & 14669.65 & 27008.00 & 45736.53 \\
0.7 & 0.15 & 1542.72 & 5903.90 & 13259.85 & 24533.18 & 41813.57 \\
0.7 & 0.30 & 1064.93 & 4119.60 & 9371.92 & 17613.44 & 30656.88 \\
0.7 & 0.45 & 672.17 & 2623.79 & 6047.15 & 11558.95 & 20605.66 \\
0.7 & 0.60 & 368.09 & 1441.11 & 3360.83 & 6539.01 & 11977.45 \\
0.8 & 0.10 & 2595.17 & 9132.28 & 19318.97 & 34115.37 & 55875.17 \\
0.8 & 0.15 & 2326.03 & 8206.84 & 17409.49 & 30841.71 & 50721.23 \\
0.8 & 0.30 & 1602.96 & 5697.51 & 12188.11 & 21807.11 & 36334.07 \\
0.8 & 0.45 & 1010.03 & 3609.45 & 7784.14 & 14071.77 & 23780.20 \\
0.8 & 0.60 & 552.13 & 1971.39 & 4278.71 & 7812.85 & 13403.33 \\
\hline
\end{tabular}

Table 6. The present relative efficiency of the proposed estimator $\hat{\pi}_{a 2}$ with respect to the direct estimator $\hat{\pi}_{D}$, i.e. $\operatorname{PRE}\left(\hat{\pi}_{a 2}, \hat{\pi}_{D}\right)$.

\begin{tabular}{rrrrrrr} 
& $\boldsymbol{P}$ & $\boldsymbol{T}$ & $\mathbf{\pi}$ & $\mathbf{0 . 5}$ \\
\cline { 3 - 7 } & 0.10 & 2595.17 & 9132.28 & 19318.97 & 34115.37 & 55875.17 \\
0.2 & 0.15 & 2326.03 & 8206.84 & 17409.49 & 30841.71 & 50721.23 \\
0.2 & 0.30 & 1602.96 & 5697.51 & 12188.11 & 21807.11 & 36334.07 \\
0.2 & 0.45 & 1010.03 & 3609.45 & 7784.14 & 14071.77 & 23780.20 \\
0.2 & 0.60 & 552.13 & 1971.39 & 4278.71 & 7812.85 & 13403.33 \\
0.3 & 0.10 & 1720.28 & 6558.88 & 14669.65 & 27008.00 & 45736.53 \\
0.3 & 0.15 & 1542.72 & 5903.90 & 13259.85 & 24533.18 & 41813.57 \\
0.3 & 0.30 & 1064.93 & 4119.60 & 9371.92 & 17613.44 & 30656.88 \\
0.3 & 0.45 & 672.17 & 2623.79 & 6047.15 & 11558.95 & 20605.66 \\
0.3 & 0.60 & 368.09 & 1441.11 & 3360.83 & 6539.01 & 11977.45 \\
0.4 & 0.10 & 1186.81 & 4767.59 & 11105.95 & 21136.70 & 36826.82 \\
0.4 & 0.15 & 1064.67 & 4296.40 & 10062.06 & 19276.07 & 33880.20 \\
0.4 & 0.30 & 735.69 & 3008.63 & 7164.63 & 14018.86 & 25371.21 \\
0.4 & 0.45 & 464.84 & 1923.46 & 4660.53 & 9336.08 & 17492.16 \\
0.4 & 0.60 & 254.83 & 1060.70 & 2613.34 & 5371.33 & 10489.57 \\
\hline
\end{tabular}




\section{TARRAY \& SINGH}

Table 7. The present relative efficiency of the proposed estimator $\hat{\pi}_{a 1}$ with respect to the direct estimator $\hat{\pi}_{a 2}$, i.e. $\operatorname{PRE}\left(\hat{\pi}_{a 1}, \hat{\pi}_{a 2}\right)$.

\begin{tabular}{|c|c|c|c|c|c|c|}
\hline \multirow[b]{2}{*}{$P$} & \multirow[b]{2}{*}{$T$} & \multicolumn{5}{|c|}{$\pi$} \\
\hline & & 0.1 & 0.2 & 0.3 & 0.4 & 0.5 \\
\hline 0.6 & 0.10 & 208.54 & 195.59 & 185.03 & 176.09 & 168.18 \\
\hline 0.6 & 0.15 & 208.46 & 195.30 & 184.41 & 175.00 & 166.41 \\
\hline 0.6 & 0.30 & 208.21 & 194.39 & 182.43 & 171.43 & 160.34 \\
\hline 0.6 & 0.45 & 207.95 & 193.42 & 180.26 & 167.31 & 152.88 \\
\hline 0.6 & 0.60 & 207.69 & 192.39 & 177.84 & 162.50 & 143.48 \\
\hline 0.7 & 0.10 & 459.60 & 400.58 & 356.70 & 322.22 & 293.55 \\
\hline 0.7 & 0.15 & 459.21 & 399.32 & 354.25 & 318.18 & 287.33 \\
\hline 0.7 & 0.30 & 458.02 & 395.41 & 346.46 & 305.13 & 266.67 \\
\hline 0.7 & 0.45 & 456.81 & 391.28 & 338.02 & 290.48 & 242.39 \\
\hline 0.7 & 0.60 & 455.56 & 386.92 & 328.82 & 273.91 & 213.48 \\
\hline 0.8 & 0.10 & 1168.00 & 923.94 & 765.56 & 652.63 & 565.52 \\
\hline 0.8 & 0.15 & 1166.27 & 919.15 & 757.19 & 640.00 & 547.37 \\
\hline 0.8 & 0.30 & 1160.98 & 904.35 & 731.03 & 600.00 & 488.89 \\
\hline 0.8 & 0.45 & 1155.56 & 888.89 & 703.20 & 556.52 & 423.53 \\
\hline 0.8 & 0.60 & 1150.00 & 872.73 & 673.53 & 509.09 & 350.00 \\
\hline
\end{tabular}

Table 8. The present relative efficiency of the proposed estimator $\hat{T}_{1}$ with respect to the proposed estimator $\hat{T}_{2}$, i.e. $\operatorname{PRE}\left(\hat{T}_{1}, \hat{T}_{2}\right)$.

\begin{tabular}{|c|c|c|c|c|c|c|}
\hline \multirow[b]{2}{*}{$P$} & \multirow[b]{2}{*}{$T$} & \multicolumn{5}{|c|}{$\pi$} \\
\hline & & 0.1 & 0.2 & 0.3 & 0.4 & 0.5 \\
\hline 0.6 & 0.10 & 155.84 & 138.02 & 129.84 & 125.15 & 122.10 \\
\hline 0.6 & 0.15 & 170.09 & 150.96 & 141.23 & 135.34 & 131.39 \\
\hline 0.6 & 0.30 & 194.19 & 177.45 & 166.92 & 159.70 & 154.44 \\
\hline 0.6 & 0.45 & 206.49 & 193.96 & 184.91 & 178.07 & 172.72 \\
\hline 0.6 & 0.60 & 214.04 & 205.47 & 198.59 & 192.95 & 188.24 \\
\hline 0.7 & 0.10 & 251.86 & 197.48 & 174.56 & 161.93 & 153.93 \\
\hline 0.7 & 0.15 & 300.32 & 236.32 & 206.82 & 189.84 & 178.81 \\
\hline 0.7 & 0.30 & 394.56 & 327.32 & 289.14 & 264.52 & 247.34 \\
\hline 0.7 & 0.45 & 449.87 & 393.59 & 356.26 & 329.68 & 309.80 \\
\hline 0.7 & 0.60 & 486.62 & 445.05 & 413.73 & 389.28 & 369.66 \\
\hline 0.8 & 0.10 & 448.61 & 311.22 & 257.83 & 229.44 & 211.83 \\
\hline 0.8 & 0.15 & 585.58 & 407.71 & 333.71 & 293.15 & 267.54 \\
\hline 0.8 & 0.30 & 901.16 & 668.74 & 552.62 & 482.98 & 436.57 \\
\hline 0.8 & 0.45 & 1125.01 & 897.50 & 764.03 & 676.28 & 614.19 \\
\hline 0.8 & 0.60 & 1293.88 & 1104.13 & 975.00 & 881.44 & 810.53 \\
\hline
\end{tabular}


Table 9. The present relative efficiency of the proposed estimator $\hat{\pi}_{a 2}$ with respect to the direct estimator $\hat{\pi}_{a 1}$, i.e. $\operatorname{PRE}\left(\hat{\pi}_{a 2}, \hat{\pi}_{a 1}\right)$.

\begin{tabular}{|c|c|c|c|c|c|c|}
\hline \multirow[b]{2}{*}{$P$} & \multirow[b]{2}{*}{$T$} & \multicolumn{5}{|c|}{$\pi$} \\
\hline & & 0.1 & 0.2 & 0.3 & 0.4 & 0.5 \\
\hline 0.2 & 0.10 & 1168.00 & 923.94 & 765.56 & 652.63 & 565.52 \\
\hline 0.2 & 0.15 & 1166.27 & 919.15 & 757.19 & 640.00 & 547.37 \\
\hline 0.2 & 0.30 & 1160.98 & 904.35 & 731.03 & 600.00 & 488.89 \\
\hline 0.2 & 0.45 & 1155.56 & 888.89 & 703.20 & 556.52 & 423.53 \\
\hline 0.2 & 0.60 & 1150.00 & 872.73 & 673.53 & 509.09 & 350.00 \\
\hline 0.3 & 0.10 & 459.60 & 400.58 & 356.70 & 322.22 & 293.55 \\
\hline 0.3 & 0.15 & 459.21 & 399.32 & 354.25 & 318.18 & 287.33 \\
\hline 0.3 & 0.30 & 458.02 & 395.41 & 346.46 & 305.13 & 266.67 \\
\hline 0.3 & 0.45 & 456.81 & 391.28 & 338.02 & 290.48 & 242.39 \\
\hline 0.3 & 0.60 & 455.56 & 386.92 & 328.82 & 273.91 & 213.48 \\
\hline 0.4 & 0.10 & 208.54 & 195.59 & 185.03 & 176.09 & 168.18 \\
\hline 0.4 & 0.15 & 208.46 & 195.30 & 184.41 & 175.00 & 166.41 \\
\hline 0.4 & 0.30 & 208.21 & 194.39 & 182.43 & 171.43 & 160.34 \\
\hline 0.4 & 0.45 & 207.95 & 193.42 & 180.26 & 167.31 & 152.88 \\
\hline 0.4 & 0.60 & 207.69 & 192.39 & 177.84 & 162.50 & 143.48 \\
\hline
\end{tabular}

Table 10. The present relative efficiency of the proposed estimator $\hat{T}_{2}$ with respect to the proposed estimator $\hat{T}_{1}$, i.e. $\operatorname{PRE}\left(\hat{T}_{2}, \hat{T}_{1}\right)$.

\begin{tabular}{|c|c|c|c|c|c|c|}
\hline \multirow[b]{2}{*}{$P$} & \multirow[b]{2}{*}{$T$} & \multicolumn{5}{|c|}{$\pi$} \\
\hline & & 0.1 & 0.2 & 0.3 & 0.4 & 0.5 \\
\hline 0.2 & 0.10 & 448.61 & 311.22 & 257.83 & 229.44 & 211.83 \\
\hline 0.2 & 0.15 & 585.58 & 407.71 & 333.71 & 293.15 & 267.54 \\
\hline 0.2 & 0.30 & 901.16 & 668.74 & 552.62 & 482.98 & 436.57 \\
\hline 0.2 & 0.45 & 1125.01 & 897.50 & 764.03 & 676.28 & 614.19 \\
\hline 0.2 & 0.60 & 1293.88 & 1104.13 & 975.00 & 881.44 & 810.53 \\
\hline 0.3 & 0.10 & 251.86 & 197.48 & 174.56 & 161.93 & 153.93 \\
\hline 0.3 & 0.15 & 300.32 & 236.32 & 206.82 & 189.84 & 178.81 \\
\hline 0.3 & 0.30 & 394.56 & 327.32 & 289.14 & 264.52 & 247.34 \\
\hline 0.3 & 0.45 & 449.87 & 393.59 & 356.26 & 329.68 & 309.80 \\
\hline 0.3 & 0.60 & 486.62 & 445.05 & 413.73 & 389.28 & 369.66 \\
\hline 0.4 & 0.10 & 155.84 & 138.02 & 129.84 & 125.15 & 122.10 \\
\hline 0.4 & 0.15 & 170.09 & 150.96 & 141.23 & 135.34 & 131.39 \\
\hline 0.4 & 0.30 & 194.19 & 177.45 & 166.92 & 159.70 & 154.44 \\
\hline 0.4 & 0.45 & 206.49 & 193.96 & 184.91 & 178.07 & 172.72 \\
\hline 0.4 & 0.60 & 214.04 & 205.47 & 198.59 & 192.95 & 188.24 \\
\hline
\end{tabular}

